\title{
Mechanical Affective Touch Therapy for Anxiety Disorders: Effects on Resting State Functional Connectivity
}

\author{
Meghan A. Gonsalves, ScM ${ }^{1,2}$; Quincy M. Beck, ScB'; \\ Andrew M. Fukuda, MD, PhD ${ }^{2,3}$; Eric Tirrell, $\mathrm{BA}^{2}$; Fatih Kokdere, $\mathrm{MD}^{2,3}$; \\ Eugenia F. Kronenberg, ScB' ${ }^{2}$; Nicolas D. ladarola, $\mathbf{M S}^{2,4}$; \\ Sean Hagberg, $\mathrm{PhD}^{5,6}$; Linda L. Carpenter, $\mathrm{MD}^{2,3}$; Jennifer Barredo, $\mathrm{PhD}^{2,3,7}$
}

\begin{abstract}
Objectives: Mechanical Affective Touch Therapy (MATT) is a safe, novel form of noninvasive peripheral nerve stimulation. Although mechanical stimulation activates nerves, we know little about its impact on psychiatric symptoms and their underlying cortical mechanisms. We examined the effects of open-label MATT on resting state functional connectivity (RSFC) and its relationship with anxiety and affective symptomatology (clinical results in separate report).

Materials and Methods: A total of 22 adults with an Axis I anxiety disorder were recruited from the community. After two initial sessions assisted by research staff, participants self-administered 20-minute sessions of MATT at home at least twice daily for four weeks. Self-report measures of mood and anxiety severity were collected at baseline, two weeks, and four weeks. Resting state functional magnetic resonance imaging was collected before the initial MATT session $(n=20)$, immediately after the first session $(n=18)$, and following four weeks of MATT $(n=14)$. Seed-based whole-brain functional connectivity analyses identified brain connectivity patterns correlated with responsiveness to MATT. Seeds were based on Neurosynth meta-analytic maps for "anxiety" and "pain" given MATT's hypothesized role in anxiety symptom amelioration and potential mechanism of action through C-tactile afferents, which play an important role in detecting pain and its affective components. Connectivity results were corrected for multiple comparisons (voxel $p<0.005$, cluster $p$-FDR $<0.05$ ).

Results: Baseline RSFC is predictive of symptom improvement with chronic MATT. Acute increases in insula connectivity were observed between mid-cingulate cortex and postcentral motor regions following the first MATT session. Chronic MATT was associated with increased connectivity between pain and anxiety regions of interest (ROIs) and posterior default mode network (DMN) regions involved in memory and self-reflection; the connectivity changes correlated with decreases in stress and depression symptoms.
\end{abstract}

Conclusions: MATT is associated with alterations in RSFC in the DMN of anxiety disorder patients both acutely and after longterm administration, and baseline RSFC is predictive of post-treatment symptom improvement.

Keywords: Anxiety disorders, magnetic resonance imaging, mechanical stimulation, neuromodulation, resting state functional connectivity \footnotetext{
Email: meghan_gonsalves@brown.edu

1 Neuroscience Graduate Program, Department of Neuroscience, Brown University, Providence, RI, USA;

2 Butler Hospital Mood Disorders Research Program and Neuromodulation Research Facility, Providence, RI, USA;

3 Department of Psychiatry and Human Behavior, Alpert Medical School, Brown University, Providence, RI, USA;

4 University of Arizona College of Medicine, Tucson, Tucson, AZ, USA.

5 AffectNeuro, Inc, Brooklyn, NY, USA;

6 Department of Neurosurgery, School of Medicine, University of New Mexico, Albuquerque, NM, USA; and

7 Center for Neurorestoration and Neurotechnology, Providence VA Medical Center, Providence, RI, USA
}

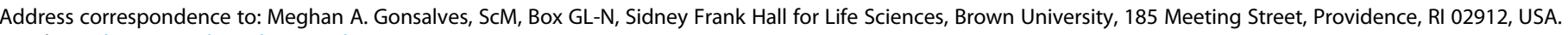

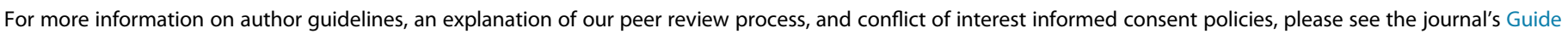
for Authors.

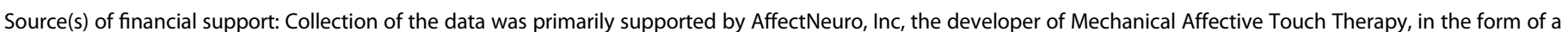

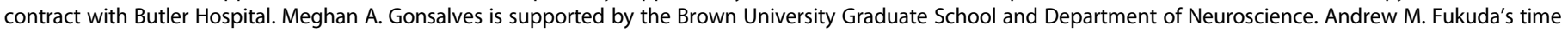

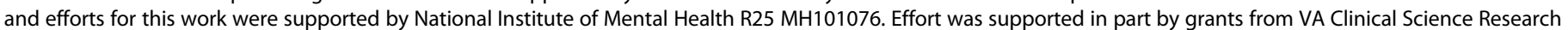

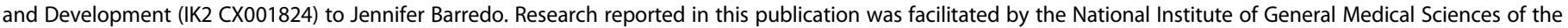

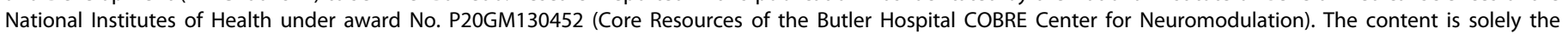
responsibility of the authors and does not necessarily represent the official views of the National Institutes of Health or the VA. 
Conflict of Interest: Collection of the data for this investigator-designed study was supported by AffectNeuro, Inc, the developer of Mechanical Affective Touch Therapy (MATT) by a grant to Butler Hospital through a contract with Butler Hospital that supported research staff effort, subject payments, and other costs associated with data collection and analysis. AffectNeuro, Inc, supplied the investigational MATT devices. Linda L. Carpenter has served as scientific advisor to Neuronetics Inc, Nexstim PLC, AffectNeuro, Inc, Neurolief Ltd, Sage Therapeutics, Sunovion, Otsuka, Neurolief, and Janssen Pharmaceuticals Inc (Antwerp, Belgium). She has received research support (to Butler Hospital) from Neuronetics Inc, Neosync Inc, Nexstim PLC, AffectNeuro, Inc, and Janssen Pharmaceuticals Inc. Sean Hagberg reports personal fees and other from AffectNeuro, Inc during the conduct of the study, and personal fees and other from AffectNeuro, Inc, outside the submitted work. In addition, Sean Hagberg has a patent $10,786,666$ issued, a patent 17/026,268 pending, a patent 16/943,204 pending, and a patent 17/035,689 pending. Jennifer Barredo received support (from AffectNeuro, Inc, funding to Butler Hospital) for consulting on functional magnetic resonance imaging analyses. The remaining authors have no conflicts of interest to report.

\section{INTRODUCTION}

Anxiety disorders are the most prevalent mental health-related illnesses in the United States, affecting approximately $19.1 \%$ of adults annually ${ }^{1}$ and $11.3 \%$ of Americans in their lifetime. ${ }^{2}$ Furthermore, disorders such as generalized anxiety disorder are universally widespread across the globe, and are associated with severe social, occupational, and physical impairment. ${ }^{3-6}$ Anxiety disorders increase risk for chronic diseases, including diabetes, cardiovascular disease, and asthma, ${ }^{7}$ and are linked with engagement in maladaptive behaviors like smoking and heavy drinking. ${ }^{8,9}$ They are also associated with greater use of disability days and decreased work productivity, placing a significant burden on the economy and health care system. ${ }^{10}$

Anxiety disorders are typically treated using a combination of psychotherapy and medication. ${ }^{11}$ Among those who complete a course of standard treatment for anxiety, symptom improvement, as measured by self-report questionnaires or clinician administered interviews, is inadequate (eg, $<50 \%$ reduction of anxiety symptoms) in one-third of patients. ${ }^{11}$ Given the substantial burden of undertreatment on patients and on society, there is a pressing need for novel anxiety disorder treatments. Noninvasive peripheral nerve stimulation is one promising alternative treatment for anxiety disorders.

All nerves that branch out of the spinal cord and brain are considered part of the peripheral nervous system. During peripheral nerve stimulation, electrical or mechanical energy is typically externally delivered to the dermal area innervated by targeted nerves or their branches, ${ }^{12}$ but in some cases (such as implanted vagus nerve stimulators), electrodes are applied directly to the nerve during a surgical procedure. A popular form of peripheral nerve stimulation is transcutaneous electrical stimulation, which reduces chronic lower back pain and acute postsurgical pain ${ }^{13,14}$; initial findings indicated it may also improve mood and anxiety disorder symptoms, ${ }^{15,16}$ perhaps through activation of afferent fibers that send signals to relevant components of the brain. Mechanical (acoustic) stimulation of peripheral nerves is another noninvasive approach and is relatively understudied regarding effects on underlying physiological systems or potential therapeutic effects. Early studies demonstrated acoustic stimulation with ultrasound $(>20 \mathrm{kHz})$ stimulates $A$-beta $(A \beta)$ peripheral nerves, ${ }^{17,18}$ and low-frequency $(<20 \mathrm{kHz})$ acoustic stimulation of somatosensory mechanoreceptors enhances proprioception. ${ }^{19}$

Mechanical Affective Touch Therapy (MATT) is a novel noninvasive peripheral mechanical nerve stimulation device developed by AffectNeuro, Inc, for treatment of anxiety disorders. The prototype of this wearable device resembles a commercially available MP3 player but delivers gentle vibratory stimulation (via insulated transducers) to small areas of skin behind each ear on the mastoid processes. The device is configured with an amplifier and piezoelectric elements or actuators (together, transducers) that enable an MP3-like signal generator to deliver gentle vibrations $(<20 \mathrm{kHz})$. Stimulation is delivered as an isochronic $10 \mathrm{~Hz}$ wave that cycles as two seconds on and two seconds off. The manufacturer's pilot data indicate that stimulation using these parameters induces a relaxed state and increased occipital alpha oscillations. ${ }^{20}$ Qualitatively, these vibrations resemble those from an electric toothbrush.

Although the mechanisms of vibratory stimulation's effect on higher level proprioception are underspecified, it may involve Piezo2 ion channels, Merkel-cell mechano-transduction, and peripheral $A \beta$ and unmyelinated $C$-tactile $(C T)$-afferent nerves. ${ }^{21}$ $\mathrm{CT}$-afferents are involved in both discriminative and affective touch. $^{22,23}$ Discriminative touch allows for the identification and detection of stimuli through vibration, texture, and pressure, whereas affective touch conveys information about the "pleasantness" of stimuli through gentle, slow stroking. ${ }^{22,23}$ Specifically, unmyelinated $\mathrm{CT}$ fibers respond to gentle vibrations and touch, firing when stroked at rates perceived as soothing or pleasurable. ${ }^{24}$ CT fibers also detect "secondary pain," which has a strong affective component, impacting attention to injury and behavioral responses to trauma. ${ }^{25,26}$ Although their main function is proprioception, myelinated $A \beta$ fibers are also implicated in the detection of neuropathic pain, such as mechanical allodynia, after damage to the nervous system. ${ }^{27,28}$ Both $A \beta$ and $C T$ afferents relay tactile and pain signals to somatosensory cortex (S1 and $S 2$ ) and emotion regions, including the insula. ${ }^{29-31}$

Integrating emotion and somatosensory signals is a core insula function essential for interoception and embodied sensation. ${ }^{32}$ Insula function, however, is often disrupted in mood and anxiety disorders and associated with hypo- or hyper-interoception. ${ }^{33,34}$ Anxiety also exacerbates hyperalgesia in people with acute and chronic pain, likely reflecting activation of overlapping pain and anxiety circuits involving the hippocampus, cingulate cortex, amygdala, and insula. ${ }^{35,36}$ Interoceptive training has been shown to reduce somatic symptoms and anxiety in nonanxious participants ${ }^{37}$ and is a cornerstone of mindfulness-based approaches to stress and anxiety reduction. ${ }^{38,39}$ However, these interventions may be less effective for those with clinical mental health struggles who may find attending to the body's internal signals aversive. ${ }^{40}$ We hypothesized that modulating somatosensory circuits via an external device may engage pain and anxiety circuits, including the insula, but be more tolerable than interoceptive training for those with distressing clinical symptoms. 
Understanding the relationship between peripheral nerve stimulation (through affective touch) and cortical function is essential for establishing the MATT device's possible mechanism of therapeutic action; mechanistic insights will be critical for later optimization of MATT. Although MATT's feasibility and efficacy are under exploration, studies investigating similar devices provide insights. Sham-controlled studies indicate transcutaneous vagus nerve stimulation (tVNS) increases magnetic resonance imaging (MRI) blood oxygen level-dependent (BOLD) activation in the insula as well as BOLD decreases in the thalamus, posterior cingulate cortex, and parahippocampal gyrus. ${ }^{41}$ These patterns have been replicated in multiple cohorts of healthy humans. ${ }^{42,43}$ Following tVNS treatment of depression, functional connectivity increases between the precuneus, orbital prefrontal cortex, and select regions of the DMN (ie, the medial prefrontal cortex, cingulate cortex, and bilateral parietal cortex), have been shown to correlate with symptom reduction. $^{44}$

The DMN is a functionally interconnected network of brain regions associated with introspection, ${ }^{45,46}$ theory of mind, $^{47}$ memory retrieval, ${ }^{48-50}$ and emotion regulation. ${ }^{51}$ Major DMN regions include bilateral lateral and medial portions of the temporal and parietal cortex, the medial prefrontal cortex, hippocampus, and parahippocampus. ${ }^{52}$ Clinically, the DMN is implicated in anxiety ${ }^{53,54}$ and mood disorders. ${ }^{55,56}$ For example, during emotion regulation, DMN BOLD signal is blunted in anxious patients compared with the activation observed in healthy controls. ${ }^{57}$ Studies measuring functional connectivity, a metric of functional cohesion between brain regions, ${ }^{58,59}$ have also found evidence of more robust connectivity between the DMN and insula in patients with heightened anxiety. ${ }^{60}$ Because external stimulation of peripheral branches of the vagus nerve can modulate brain activity in both DMN and insula ${ }^{41-43}$ in healthy individuals, mechanical stimulation with MATT may hold potential for anxiety treatment, given the involvement of both in anxiety and pain circuits. ${ }^{61}$ Although certain brain regions have been implicated in anxiety, no consensus morphometric biomarkers for anxiety disorders have been established. ${ }^{62,63}$ MATT also represents an opportunity to explore potential volumetric biomarkers for anxiety, ${ }^{64}$ their responsiveness to peripheral nerve stimulation, and relationships with RSFC.

We used RSFC to investigate the relationship between a novel form of peripheral nerve stimulation via affective touch for anxiety and cortical function. Acute and chronic RSFC effects of MATT in pain and anxiety circuits were evaluated in a small, heterogeneous sample of adults with various anxiety disorders in an open-label trial. We hypothesized that acute changes in connectivity and neural predictors of treatment response would localize to the DMN. Moreover, we anticipated that changes in DMN connectivity would correspond to symptom changes across treatment. We explored MATT's effects on morphometrics, and how such changes relate to RSFC. We also explored potential links between connectivity and MATT-associated changes in interoception.

\section{MATERIALS AND METHODS}

\section{Study Overview}

This was an open-label pilot trial designed to explore clinical effect and potential mechanisms of action of a novel investigational device for anxiety disorders. All participants received active MATT using the research prototype device. Resting state functional MRI and structural MRI scans were obtained at three time points: immediately before first application of MATT stimulation (T1); immediately after 15 minutes of MATT stimulation (T2); and after completion of a four-week course of daily MATT sessions (T3). We compared RSFC data from $\mathrm{T} 1$ and $\mathrm{T} 2$ to evaluate whether acute changes in functional MRI connectivity correspond to acute changes in the electroencephalogram (EEG) signal that we observed during device/parameter development. We compared RSFC between T1 and T3 to identify connectivity changes following the full course of treatment. Clinical assessments of anxiety, depression, and stress symptoms were collected at baseline, week two midpoint, and study endpoint. Interoception was measured at baseline and study endpoint.

\section{Participants}

Adult outpatients $(N=22)$ aged 18 to 65 years with a current Axis I anxiety disorder ${ }^{65}$ were recruited from the local community, enrolled, and initiated treatment. Written informed consent was obtained on forms approved by the Butler Hospital Institutional Review Board. Participants were required to be medication-free or on a stable regimen of psychotropic medications (ie, not started a new medication or changed doses of ongoing medications) for 30 days before the baseline visit and throughout the duration of their study participation. Participants were excluded if they had been psychiatrically hospitalized or had attempted suicide within the previous six months, had MRI safety contraindications, or were diagnosed with significant neurological conditions or other severe medical conditions that could limit compliance with study procedures. All but one of 22 enrolled study participants completed the baseline MRI session (T1) and had at least one symptom assessment after initiating treatment; a subset $(n=17)$ completed post-treatment MRI scans (T3). In total, 20 participants were included in T1 RSFC analyses (one participant from the original 21 scanned was excluded because of motion artifacts); 18 participants were included in T1-T2 analyses (two were excluded because of motion artifacts from the original 20); and 14 participants were included in the T1-T3 analyses (17 completed the scan, but three were removed because of motion artifacts). Differences in clinical symptoms and demographics among the three MRI analysis groups were measured using one-way repeated measures ANOVAs.

\section{Diagnostic and Symptom Assessments}

Medical/neurological health histories and current medication regimens were reviewed and recorded at baseline. Confirmation of current Axis I anxiety disorder was done with the Mini-International Psychiatric Interview (MINl; Table 1, baseline participant demographics). ${ }^{66}$ At least a moderate level of baseline anxiety (Generalized Anxiety Disorder, seven-item scale $[G A D-7]^{67}$ total $\geq 10$ ) was required for eligibility. Participants completed a battery of selfreport scales at pre-treatment baseline, week two, and week four (or early termination). We selected the GAD-7 total score, which covers a spectrum of anxiety symptoms, as our primary outcome measure. We felt this was appropriate given that we had no strong a priori expectation that MATT's anxiolytic effects would be specific to one anxiety subdomain. We did, however, collect a number of secondary outcome measures to permit initial exploration of symptom domain effects: the Perceived Stress Scale (PSS), ${ }^{68}$ the Beck Depression Inventory (BDI), ${ }^{69}$ the Depression, Anxiety, and Stress Scale (DASS), ${ }^{70}$ and the Multidimensional Assessment of Interoceptive Awareness (MAIA). ${ }^{71}$ We analyzed total scores from the DASS, MAIA, BDI, and PSS, as well as DASS subscale scores for 
Table 1. Descriptive and Clinical Data for All Participants $(N=20)$ in Imaging Analyses (T1)

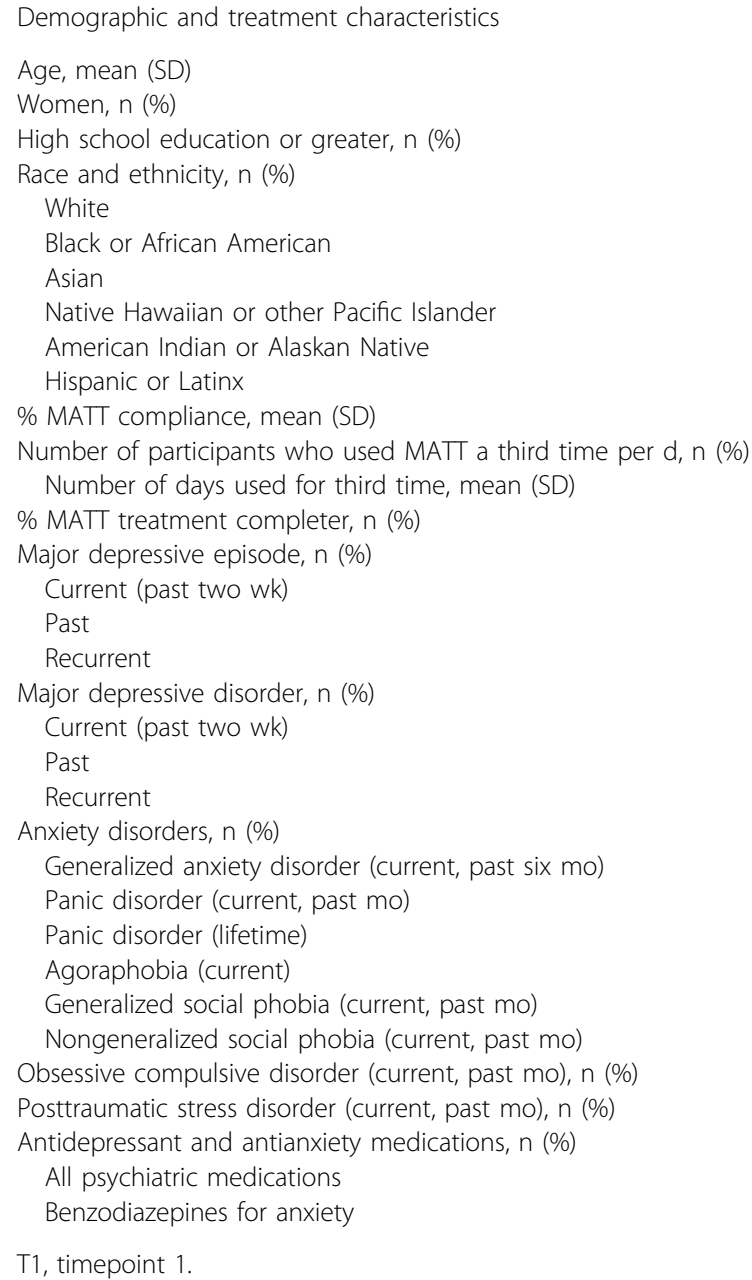

T1, timepoint 1.

depression (DASS Depression), anxiety (DASS anxiety), and stress (DASS stress). See Table 2 for scores on symptom assessments in the total sample $(n=20)$.

\section{Treatment}

The MATT device delivers gentle mechanical stimulation behind each ear via small $(30 \mathrm{~mm}$ ) piezoelectric disks that are mounted on a headset. The power and signal are generated from a modified MP3 player that effectively "plays" the signal converted to vibration. The research team helped participants identify the optimal stimulation intensity-the just-perceivable vibrational intensity that was consistently detectable but not uncomfortable. The device's settings range from 0 to 20 in arbitrary units. At baseline, participants were instructed to put on the device and turn it to a high, perceivable level (eg, 18). They then systematically turned the intensity down by one level until they reached a point where they could no longer feel the vibrations. The final intensity was set at one level above this point. Details regarding device development, parameter selection, and safety are reported in the device patent. ${ }^{20}$

The first and second stimulation sessions were administered under the observation of research staff at the baseline MRI visit and baseline EEG visit, respectively. Participants were then instructed to selfadminister MATT at home or in other naturalistic settings twice a day
(20 minutes per session) for four weeks (28 days), with an option to use MATT a third time each day if needed for acute anxiety or during anxiety-provoking situations. Treatment length and parameters were selected based on feasibility and initial pilot data. ${ }^{20}$ Participants wore MATT for 15 instead of 20 minutes during the baseline MRI visit because of time constraints at the scanner. Treatment compliance was defined as the percentage of completed self-administered MATT sessions out of the prescribed number of sessions (two sessions per day for 28 days). Participants who completed the week four symptom assessments and post-treatment MRI were classified as completers. Participants who were treated with MATT but who did not complete the final week four visit were included in the intent-to-treat (ITT) sample; last observation carried forward (LOCF) scores were used when visit four data were not collected. Of the 20 participants included in analyses described here, 15 (75\%) were completers and the other five $(25 \%)$ discontinued before visit four. All individuals in the ITT sample were included in the T1 analyses regardless of completion status.

\section{MRI Data Collection and Preprocessing}

All brain scan procedures took place at the Brown University MRI Facility. The first two scans occurred during a baseline visit, and the final scan during an endpoint visit (after four weeks of MATT) using a Siemens 3T MRI Scanner (Erlangen, Germany) and a 64-channel 
Table 2. Clinical Measure Scores by Scale for All Participants $(n=20)$ in Imaging Analysis (T1).

\begin{tabular}{|c|c|}
\hline Scale & Mean (SD) \\
\hline \multicolumn{2}{|l|}{ GAD-7 } \\
\hline Baseline & $14.45(2.04)$ \\
\hline LOCF & $8.90(5.16)$ \\
\hline$\%$ change: baseline-LOCF & $-36.82(36.85)$ \\
\hline \multicolumn{2}{|l|}{ PSS } \\
\hline Baseline & $36.65(5.51)$ \\
\hline LOCF & $28.45(8.21)$ \\
\hline$\%$ change: baseline-LOCF & $-22.57(17.24)$ \\
\hline \multicolumn{2}{|l|}{ BDI } \\
\hline Baseline & $30.30(7.92)$ \\
\hline LOCF & $18.15(12.91)$ \\
\hline$\%$ change: baseline-LOCF & $-40.93(37.18)$ \\
\hline \multicolumn{2}{|l|}{ DASS } \\
\hline \multicolumn{2}{|l|}{ Total } \\
\hline Baseline & $57.30(20.74)$ \\
\hline LOCF & $35.85(26.89)$ \\
\hline$\%$ change: baseline-LOCF & $-37.16(36.84)$ \\
\hline \multicolumn{2}{|l|}{ Depression } \\
\hline Baseline & $20.35(9.37)$ \\
\hline LOCF & $12.95(11.98)$ \\
\hline$\%$ change: baseline-LOCF & $-39.85(42.85)$ \\
\hline \multicolumn{2}{|l|}{ Anxiety } \\
\hline Baseline & $14.20(7.41)$ \\
\hline LOCF & $8.80(6.70)$ \\
\hline$\%$ change: baseline-LOCF & $-26.12(58.74)$ \\
\hline \multicolumn{2}{|l|}{ Stress } \\
\hline Baseline & $22.75(7.71)$ \\
\hline LOCF & $14.10(10.12)$ \\
\hline$\%$ change: baseline-LOCF & $-36.45(34.34)$ \\
\hline \multicolumn{2}{|l|}{ MAIA total } \\
\hline Baseline & $2.45(0.32)$ \\
\hline LOCF & $2.65(0.61)$ \\
\hline$\%$ change: baseline-LOCF & $8.12(0.24)$ \\
\hline
\end{tabular}

head coil. Immediately before the first MATT stimulation, a structural T1-weighted image (TE $=1.69 \mathrm{msec}, \mathrm{TR}=2530 \mathrm{msec}, \mathrm{FOV}=$ $256 \mathrm{~mm}^{2}, 1 \mathrm{~mm}^{3}$ ) was collected. Resting state echoplanar images were then acquired for ten minutes via whole-brain continuous imaging in the transverse plane (TE $=30 \mathrm{msec}, \mathrm{TR}=1000 \mathrm{msec}$, FOV $=192 \mathrm{~mm}^{2}, 2 \mathrm{~mm}^{3}, 588$ volumes, multiband factor $=4$, slice thickness $=2 \mathrm{~mm}^{3}$, spacing between slices $=2 \mathrm{~mm}^{3}, 60$ slices). Participants were instructed to lie still and focus their gaze on a display screen showing a white crosshair in the middle of a black foreground during rest scans. Participants were removed from the scanner and had their first MATT stimulation session in a separate room, after which they returned to the scanner for additional T1weighted structural and resting state scans. Following the fourweek course of MATT and final clinical assessments, structural and resting state scans were repeated.

All MRI data preprocessing steps were executed with the CONN Toolbox $^{72}$ (https://web.conn-toolbox.org). Standard MRI preprocessing steps included slice-time correction, motion estimation and realignment, normalization of images to Montreal Neurological Institute (MNI)-152 Atlas space, and spatial smoothing with a $6 \mathrm{~mm}$ full-width half-max gaussian kernel. Additional functional connectivity preprocessing steps were applied to reduce the contribution of nonneuronal signals and motion on functional connectivity. ${ }^{73,74}$
The Anatomical CompCor method ${ }^{75}$ modeled nonneuronal signals: five principle components were computed from white matter and cerebrospinal fluid BOLD time courses. These components were then regressed from subjects' preprocessed data along with six estimated motion parameters (three translational, three rotational), their first temporal derivatives, and the linear trend. Residuals were then band-pass filtered (high-pass filtering: $0.008 \mathrm{~Hz}$; low-pass filtering: $0.09 \mathrm{~Hz}$ ) before first-level modeling.

\section{Subject-Level Seed-to-Voxel Analyses}

Functional regions of interest (ROls) or functional connectivity "seeds" were based on construct maps for "pain" and "anxiety" in Neurosynth (https://neurosynth.org/). Neurosynth" is a metaanalytic tool that generates functional connectivity maps for lexical terms and cognitive processes. The construct "anxiety" was chosen for ROI extraction given MATT's hypothesized role in ameliorating symptoms across a number of anxiety disorders; "pain" was also selected given MAT's potential mechanism of action through CT-afferents. These afferents play an important role in detecting pain and corresponding affective states. This method allowed us to remain objective in ROI selection and solely use empirically based seeds.

To define our ROls, we thresholded the map of each term using a minimum $z$ score and extracted clusters of spatially contiguous voxels. Thresholding the "anxiety" map at $z$ scores $>5$ yielded two ROIs centered on the amygdala in each hemisphere. We used a more stringent threshold ( $z$ scores $>7$ ) for the "pain" ROls to improve cluster separation. This produced bilateral clusters in the anterior insula and thalamus, and a mid-cingulate ROI crossing the sagittal midline.

For each seed, we constructed a whole-brain voxel-wise map of correlations with the seed's BOLD time-course. These subject-level maps underwent Fisher R-to-Z transformation to improve conformation to the assumptions of generalized linear models. The seed maps were entered into second-level analyses of covariance (ANCOVA; indicated subsequently in the text).

\section{Second-Level Hypothesis Testing and Cross-Validation}

Second-level models were constructed to 1) identify pre-treatment connectivity patterns predictive of subsequent treatment outcomes; 2) localize acute connectivity changes immediately after MATT; 3) determine whether acute connectivity changes predicted treatment outcome, and 4) identify post-treatment correlates of symptom improvement. All model results were evaluated using a dual thresholding procedure with an uncorrected cluster-forming voxel height threshold of $p<0.005$ and cluster-level multiple comparisons correction at $p$-FDR $<0.05$. A leave-one-out crossvalidation analysis was performed for all significant clusters for an additional level of stringent control. Briefly, on each iteration, models were reestimated leaving one subject out, and a parameter estimate (beta weight) was generated for the left-out subject based on this model. To determine if a cluster cross-validated, we submitted these estimated weights to a two-tailed $t$-test against the mean with alpha set at $p<0.05$. Two-tailed $t$-tests were selected as a stringent test of cluster cross-validation. We also excluded clusters if they were present in $<80 \%$ of cross-validation masks. Only those results that survived leave-one-out crossvalidation are presented in this report.

To identify clusters predictive of treatment outcomes, we constructed continuous variables corresponding to clinical assessment 
scale scores at study endpoint (or LOCF). We then entered seed maps from the pre-treatment imaging session (T1) into an ANCOVA model evaluating the between-subjects' effect of endpoint scores after covarying for baseline symptom severity.

To localize acute effects of MATT, we compared T1 seed maps with those collected immediately after MATT delivery (T2), evaluating within-subject change after covarying for baseline symptom scores. To localize brain regions where acute changes in functional connectivity were associated with subsequent improvement in clinical symptoms, we tested the between-subjects effect of posttreatment symptom change with session $(\mathrm{T} 1>\mathrm{T} 2)$ as the withinsubjects factor. To account for baseline differences in symptom severity, clinical and interoceptive changes associated with MATT were operationalized as percent change in scale score (GAD-7, DASS Total, DASS Depression, DASS Anxiety, DASS Stress, PSS, BDI, and MAIA Total) from baseline to endpoint. LOCF values were applied in cases where a participant terminated before week four assessments.

To identify functional correlates of clinical improvement with chronic MATT, we tested the significance of the between-subjects effect of symptom change on pre-treatment versus post-treatment (T1-T3) seed-to-voxel connectivity.

\section{Morphometry Analyses}

Freesurfer (version 5.3, http://surfer.nmr.mgh.harvard.edu/) software was used to explore the relationship between functional connectivity changes associated with MATT response and brain structure. ${ }^{77}$ Subjects' structural images from the T1 and T3 sessions were preprocessed using the "fsrecon-all" routine. Steps included skull stripping, volumetric labeling, intensity normalization, tissue parcellation, registration to Freesurfer's default spherical atlas ("fsaverage"), surface extraction, and cortical labeling. For complete technical details of Freesurfer preprocessing see the following references. ${ }^{78-84}$ We examined cortical thickness in the insula and mid-cingulate ${ }^{85}$ and subcortical volumes in MNI space in the left thalamus and amygdala. Metrics were calculated and extracted by Freesurfer. Volume estimates were adjusted to account for differences in brain volume. We then used SPSS (version 25, IBM Corporation, Armonk, NY) to compute correlations between subjectlevel morphometry values and beta coefficients from the analysis of functional connectivity changes post-MATT. For this exploratory analysis, significance was determined using a more liberal onetailed $p<0.05$ statistical threshold for these bivariate correlations.

\section{RESULTS}

\section{Participant Demographics and Clinical Outcomes}

No significant differences in clinical symptoms and demographics were found among the three MRI analysis groups $(p>0.05$; Supplementary Tables 1 and 2). Most participants with imaging data ( $n=20$ ) were taking stable doses of prescribed antianxiety/antidepressant medications ( $n=14 ; 70 \%$ ); the remaining participants ( $n=6 ; 30 \%$ ) were not. Of those on medications, four were on stable doses of benzodiazepines. Supplementary Table 1 contains a breakdown of clinical and demographic information for each MRI analysis group. Mean treatment compliance was high $(74.50 \%$ of prescribed doses) for participants included in imaging analyses. A significant relationship was found between treatment compliance and percent change on the BDI ( $r=0.60, p<0.01, n=20)$, but compliance was not significantly correlated with any other symptom measure. Further details regarding MATT compliance, as-needed MATT dose, and study completion appear in Table 1 and Supplementary Table 1. Among completers $(n=15)$, paired $t$-tests showed MATT was associated with significant reductions $(p<0.01)$ in mean scores for all symptom measures (GAD-7, PSS, BDI, DASS Total, DASS Anxiety, DASS Stress, and DASS Depression) and a significant increase in MAIA Total scores (all $p<0.05$ ) from baseline to study endpoint. See Table 2 for clinical measure scores by scale for all participants at baseline and LOCF. Symptom and interoceptive scale data for each MRI analysis group appear in Supplementary Table 2.

\section{Predictors of Treatment Outcomes}

Stronger positive connectivity between pain and anxiety regions to the DMN was generally predictive of greater clinical improvement at treatment endpoint. Functional connectivity of the left amygdala to DMN clusters in both the right and left lateral temporal cortex was negatively correlated with LOCF GAD-7 scores (both cross-validated $p<0.005$ ). Similarly, functional connectivity of left anterior insula to left posterior supramarginal gyrus was negatively correlated with post-treatment PSS scores (cross-validated $p<0.01$; Fig. 1), though this part of the parietal cortex is implicated in executive control networks, rather than DMN. Stronger positive connectivity between the cingulate pain $\mathrm{ROI}$ and the left precuneus (DMN) was also associated with post-treatment improvement in DASS Total composite scores (cross-validated $p<0.001$ ). Closer examination of DASS by subscale indicated that this relationship was driven by the reductions in the DASS Stress subscale scores (Fig. 1). All findings remained significant after covariance for symptom severity at baseline and for treatment adherence (represented as percent completed MATT sessions based on participant daily logs). See Table 3 for additional cluster information.

\section{Acute Changes in Functional Connectivity}

We observed increases in right anterior insula functional connectivity immediately after the initial session of MATT stimulation (Table 4). Weak positive connectivity between the right insula seed and right middle cingulate cortex and left precentral gyrus observed at baseline became stronger after MATT (cross-validated $p<0.001$; Fig. 2). Before stimulation, right insula and left precentral time courses were anticorrelated, but they became positively correlated after stimulation (cross-validated $p<0.005$ ). Post hoc tests indicated that these connectivity relationships remained significant when baseline clinical symptom severity scores (GAD-7, BDI, PSS, DASS Total, DASS Stress, DASS Anxiety, DASS Depression, and MAIA Total) were included as model covariates.

\section{Functional Connectivity and Post-Treatment Symptom Change}

Increases in positive functional connectivity between the cingulate cortex and the left anterior supramarginal cortex after MATT completion were correlated with decreases in DASS Total scores (cross-validated $p=0.05$ ). Additional testing conducted within DASS subscales indicated that this connectivity relationship was associated with changes in depression (cross-validated $p<0.05$ ) and stress (cross-validated $p=0.06$ ), but not with changes on the anxiety subscale (Fig. 3, Table 5).

\section{Exploratory Structure-Function Correlations}

Our morphometry results preliminarily indicate that the thickness of cortical structures in circuits associated with pain may 


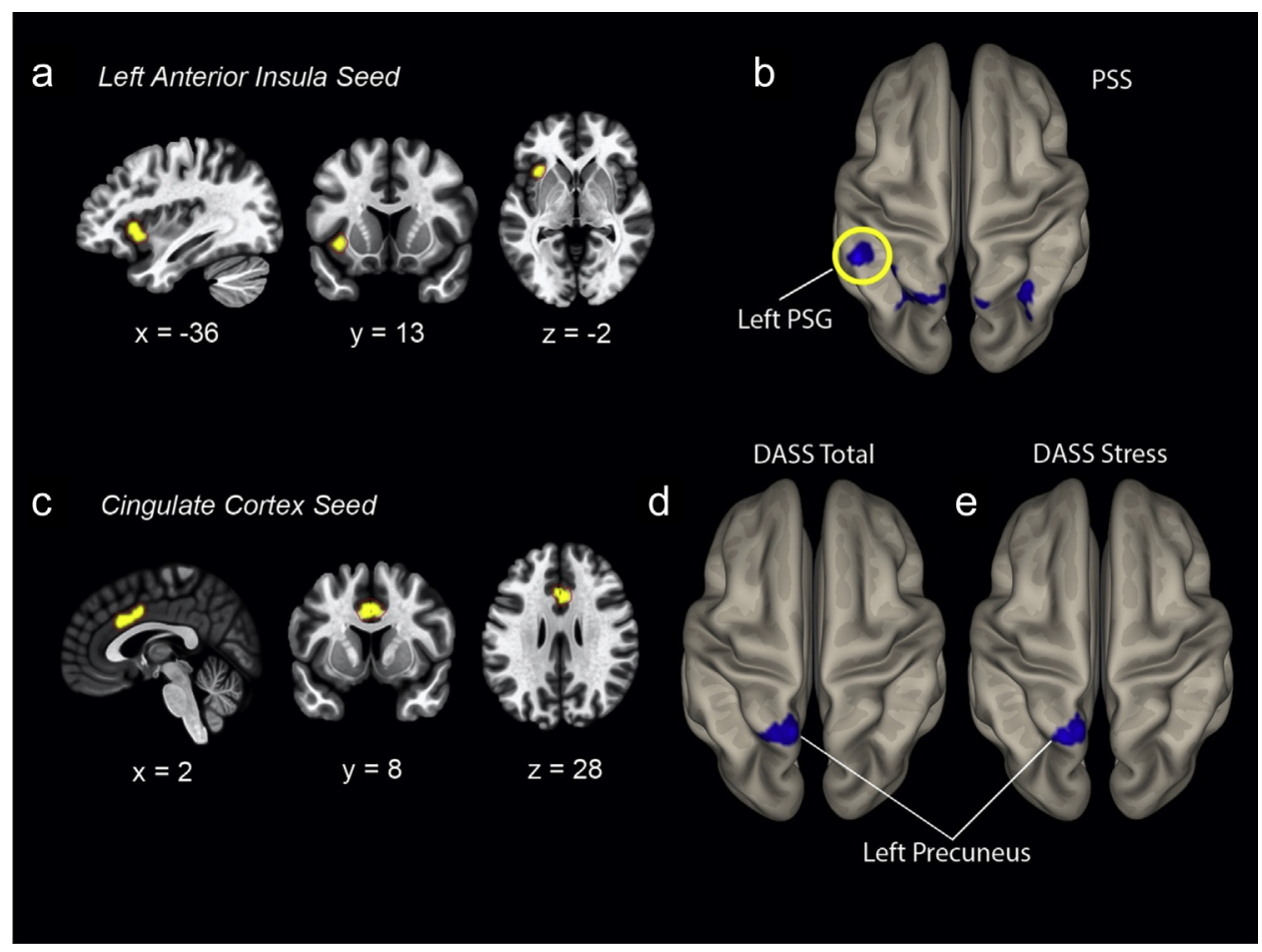

Figure 1. Pre-treatment functional connectivity seed-to-cluster pairs associated with post-MATT symptom improvement (T1). a. Sagittal, coronal, and axial representations of the left anterior insula seed derived from Neurosynth. b. Superior view of functional connectivity of the left anterior insula seed (a) to the left posterior supramarginal gyrus (PSG) cluster (circled in yellow) negatively correlated with post-treatment PSS scores (cross-validated $p<0.01$ ). c. Sagittal, coronal, and axial representations of the bilateral cingulate cortex seed derived from Neurosynth. d. Superior view of functional connectivity of the cingulate cortex seed (c) to the left precuneus as associated with post-treatment improvement in total DASS scores (cross-validated $p<0.001$ ). e. Superior view of the same pattern of functional connectivity as seen in d however, this is associated with post-treatment improvement in DASS Stress scores (cross-validated $p<0.001$ ). All neuroanatomical images were derived using CONN toolbox. [Color figure can be viewed at www.neuromodulationjournal.org]

influence functional response to MATT. Anterior cingulate-toanterior supramarginal functional connectivity-associated with DASS Depression scores post-MATT-was negatively correlated with cortical thickness in both the right insula $\left(r_{(14)}=-0.48\right.$, $p<0.05)$ and the left cingulate $\left(r_{(14)}=-0.51, p<0.05\right)$.

\section{DISCUSSION}

This study examines the impact of noninvasive transcutaneous mechanical transduction on brain connectivity. We examined the relationship between changes in mood/anxiety symptoms and
RSFC in individuals receiving peripheral nerve stimulation with the MATT device-a novel acoustic, noninvasive mechanical stimulation device designed to treat anxiety disorders. Broadly, these preliminary results indicate that 1) MATT may be associated with acute modulation of pain and anxiety networks, and 2) that changes in connectivity between pain and internal mentation networks may influence clinical response to mechanical stimulation.

As hypothesized, more robust pre-treatment functional connectivity between pain and anxiety regions and the DMN predicted superior treatment outcomes. Previous studies found that stronger connectivity between these networks is associated with anxiety

Table 3. Pre-Treatment Functional Connectivity Relationships Associated With Post-MATT Symptom Improvement (T1).

\begin{tabular}{|c|c|c|c|c|}
\hline Scale & Seed-to-cluster pair & Peak MNI coordinate & Cluster size & Cluster $p$-FDR \\
\hline \multirow[t]{2}{*}{ GAD-7 } & L. amygdala-R. AMTG & $+60-08-12$ & 280 & $<0.0001$ \\
\hline & L. amygdala-L. planum polare & $-48-02-20$ & 98 & $<0.05$ \\
\hline PSS & L. anterior insula-L. PSG & $-50-42+48$ & 317 & $<0.0001$ \\
\hline DASS total & L. thalamus-R. MTP & $+44+20-38$ & 109 & $<0.05$ \\
\hline DASS anxiety & L. anterior insula-L. PSG & $-50-42+36$ & 335 & $<0.0001$ \\
\hline DASS stress & L. amygdala-L. STG & $-44-14+00$ & 135 & $<0.05$ \\
\hline
\end{tabular}

AMTG, anterior middle temporal gyrus; FDR, false-discovery rate; L, left; MTP, medial temporal pole; PMTG, posterior middle temporal gyrus; PSG, posterior supramarginal gyrus; R, right; STG, superior temporal gyrus; T1, timepoint 1. 
Table 4. Clusters Associated With Acute Changes in RSFC After Initial MATT Administration (T1-T2)

\begin{tabular}{|c|c|c|c|c|}
\hline Scale & Seed-to-cluster pair & Peak MNI coordinate & Cluster size & Cluster $p$-FDR \\
\hline PSS & R. anterior insula-L. PG & $-40-26+58$ & 411 & $<0.000001$ \\
\hline \multirow[t]{2}{*}{$\mathrm{BDI}$} & R. anterior insula-L. PG & $-38-28+60$ & 356 & $<0.00001$ \\
\hline & R. anterior insula-R. MCG & $+06-06+38$ & 225 & $<0.001$ \\
\hline DASS depression & R. anterior insula-L. PG & $-32-30+54$ & 380 & $<0.00001$ \\
\hline DASS anxiety & R. anterior insula-R. MCG & $+06-06+38$ & 193 & $<0.001$ \\
\hline \multirow[t]{2}{*}{ DASS stress } & R. anterior insula-L. PG & $-38-28+60$ & 381 & $<0.00001$ \\
\hline & R. anterior insula-R. MCG & $+06-06+38$ & 214 & $<0.001$ \\
\hline \multirow[t]{2}{*}{ MAIA total } & R. anterior insula-L. PG & $-38-28+60$ & 381 & $<0.000001$ \\
\hline & R. anterior insula-R. MCG & $+06-06+38$ & 205 & $<0.001$ \\
\hline
\end{tabular}

FDR, false-discovery rate; L, left; MCG, middle cingulate gyrus; PG, precentral gyrus; R, right; T1, timepoint 1; T2, timepoint 2.

severity. ${ }^{86,87}$ In this study, we found that stronger inter-network connectivity predicted superior reduction in anxiety and stress symptoms. Specifically, stronger connectivity between the amygdala ("anxiety" seed) and the lateral temporal cortex at baseline was linked to greater anxiety reduction following a four-week course of MATT. Similarly, stronger functional connectivity between the insula ("pain" seed) and the precuneus (DMN) was associated with larger decreases in stress. Although DMN regions generally contribute to internally focused cognition, functional fractionations of this network link lateral temporal DMN to social cognition, and midsagittal DMN to affect and memory. ${ }^{88}$ We speculate that stronger connectivity between anxiety regions and the DMN may be associated with memory capacity through increased crossnetwork functional integration. 89,90

In contrast to our a priori expectations based on observations from $\mathrm{tVNS}^{41}$ we did not observe functional connectivity changes

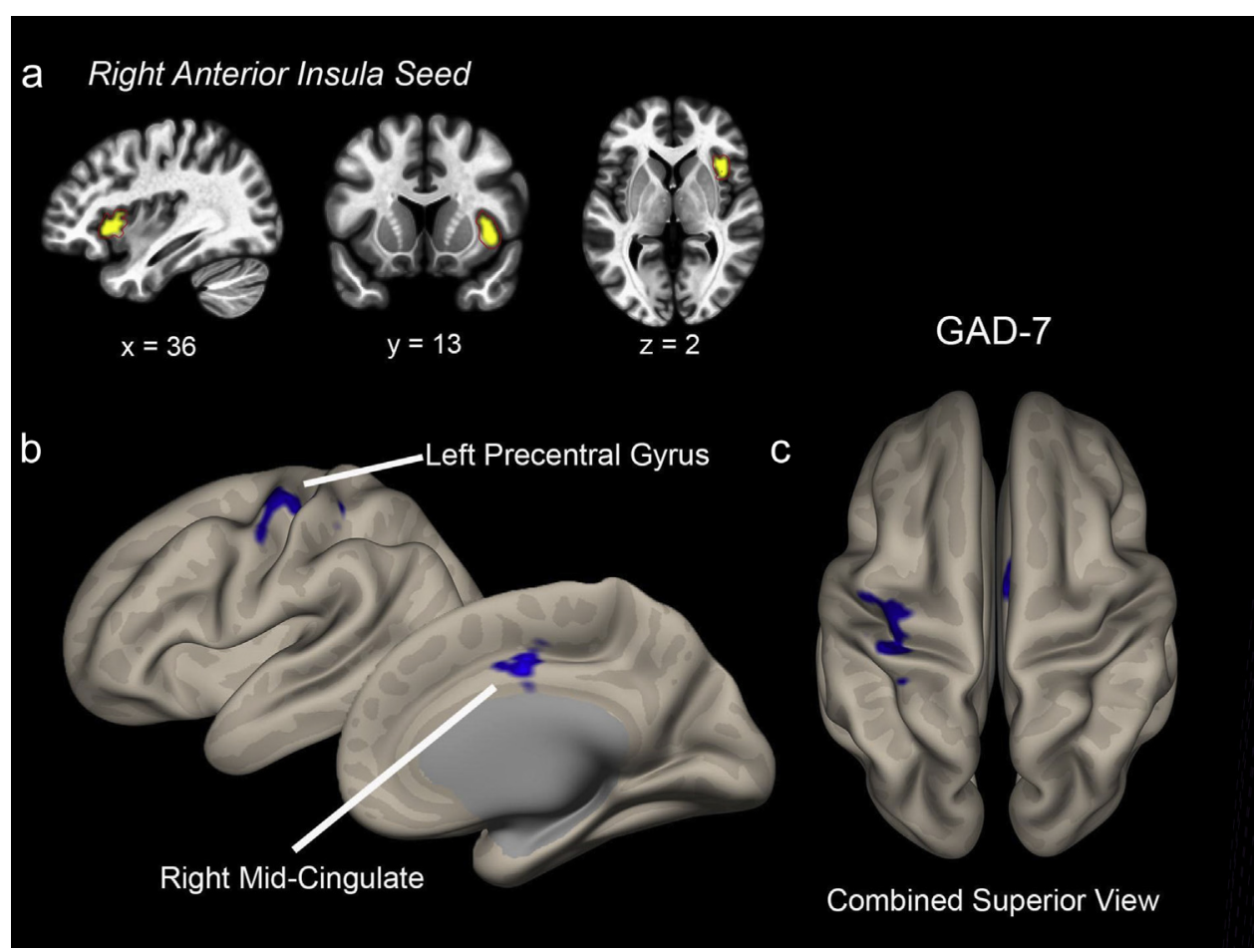

Figure 2. Seed-to-cluster pairs associated with acute changes in RSFC after initial MATT administration (T1-T2). a. Sagittal, coronal, and axial representations of the right anterior insula seed derived from Neurosynth. b. Positive connectivity between the right anterior insula seed (a) and the left precentral gyrus (lateral view) and right mid-cingulate cortex (medial view) while controlling for baseline GAD-7 scores (cross-validated $p<0.001$ ). c. A combined superior view of the left precentral gyrus and right mid-cingulate cortex clusters while controlling for baseline GAD-7 scores. All neuroanatomical images were derived using CONN toolbox. [Color figure can be viewed at www.neuromodulationjournal.org] 


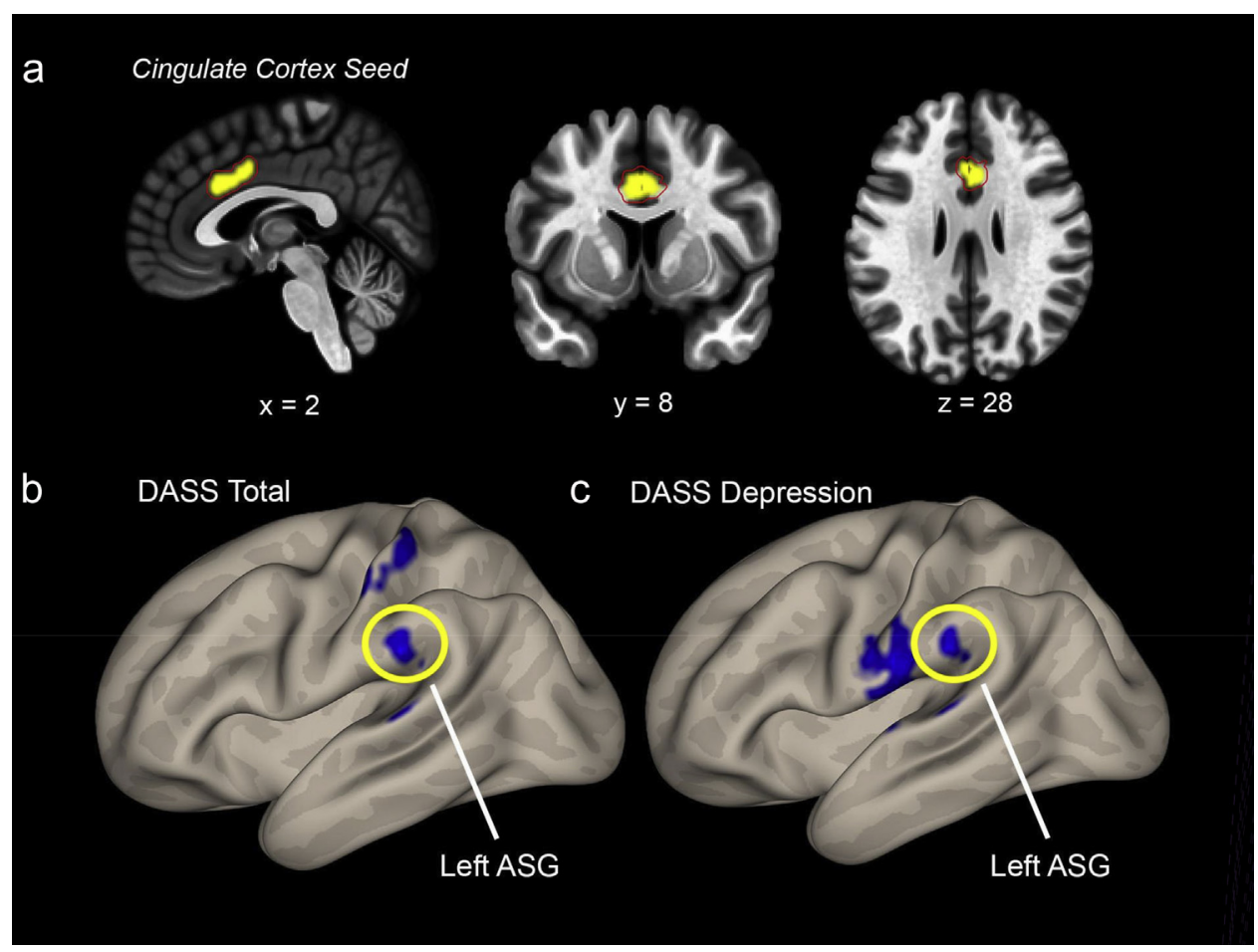

Figure 3. Seed-to-voxel functional connectivity clusters associated with percent change in symptom improvement after MATT treatment (T1-T3). a. Sagittal, coronal, and axial representations of the bilateral cingulate cortex seed derived from Neurosynth. b. Lateral view of functional connectivity between the cingulate cortex seed (a) and the left anterior supramarginal gyrus (ASG) correlated with decreases in total DASS scores after MATT completion (cross-validated $p=0.05$ ). c. Lateral view of the same pattern of functional connectivity as seen in $b$, however, this is correlated with decreases in DASS Depression scores after MATT completion (cross-validated $p=0.06$ ). All neuroanatomical images were derived using CONN toolbox. [Color figure can be viewed at www.neuromodulationjournal.org]

between pain and anxiety networks to DMN after a single (initial) MATT administration. Instead, we observed acute increases in insula connectivity to pain and motor regions, including the midcingulate and postcentral cortex. Although the insula is associated with pain, ${ }^{91}$ it is also part of a broader network inclusive of midcingulate $^{92,93}$ and postcentral cortex ${ }^{94}$ involved in salience monitoring $^{95}$ and embodied sensation. ${ }^{96,97}$ We surmise that the observed pattern of acute connectivity increases could reflect the engagement of salience or haptic/pain monitoring in relation to MATT stimulation.

Finally, we observed the anticipated correlation of changes in connectivity and symptoms following chronic MATT for pain seeds. Increases in mid-cingulate connectivity with the lateral subnetwork of the DMN were correlated with reductions in depression and stress scores on the DASS at study endpoint. This prediction, however, did not hold for our anxiety seeds. This null finding may reflect our use of a small, naturalistic, and heterogeneous sample and inclusion criterion favoring the type of anxiety symptoms measured by the
GAD-7 (primarily cognitive symptoms) over other types of anxiety symptoms (eg, somatic). Alternatively, the latency to symptom response may differ across clinical symptoms, or MATT may modify different networks across the course of treatment. To wit, we observed changes in pain network connectivity to salience and interoception regions acutely, whereas post-treatment pain connectivity effects localized to DMN. Similarly, associations between changes in MAIA scores and RSFC were also non-significant. While this may indicate that MATT's effect on interoception is limited, changes in interoceptive awareness may develop over a longer time window than the four weeks between pre- and post-MATT intervention (ie, four weeks). Interoceptive awareness, as measured by the MAIA, has typically been shown to significantly increase with interventions that span a longer period of time (eg, three months ${ }^{98}$ ).

While exploratory, we note that our preliminary structural results also highlight the centrality of pain and salience circuits to MATT response. Our early evidence indicates that cortical thickness in

Table 5. Seed-to-Voxel Functional Connectivity Clusters at T1-T3 Associated With \% Change in Symptom Improvement Upon the End of MATT Treatment.

\begin{tabular}{|c|c|c|c|c|}
\hline Scale & Seed-to-cluster pair & Peak MNI coordinate & Cluster size & Cluster $p$-FDR \\
\hline $\begin{array}{l}\text { DASS total } \\
\text { DASS depression }\end{array}$ & $\begin{array}{l}\text { Cingulate cortex-L. ASG } \\
\text { Cingulate cortex-L. ASG }\end{array}$ & $\begin{array}{l}-66-34+24 \\
-66-34+24\end{array}$ & $\begin{array}{l}186 \\
168\end{array}$ & $\begin{array}{l}<0.001 \\
<0.01\end{array}$ \\
\hline
\end{tabular}


these circuits may influence how the brain functionally responds to treatment with MATT. Although this analysis was conducted in a limited subsample of participants and must be regarded as preliminary, greater exploration of structure-function relationships may reveal new in-roads into personalized medicine approaches to treating anxiety. The utility of morphometry should be explored in future larger scale randomized control trials.

\section{Limitations}

Several limitations of the current study must be noted. First, this preliminary study used an open-label design without a sham control condition. It remains to be seen if results will replicate in a blinded, randomized controlled trial. We also note that our sample size was small and heterogeneous in terms of anxiety disorders, and despite cross-validations, these imaging results should be regarded as preliminary until replicated in a larger sample. Our small sample size also limits our power to detect small-to-medium effect sizes, and we acknowledge the potential for Type II error. ${ }^{99}$ In addition, our primary measure of anxiety, the GAD 7-item scale, had fewer questions and thus less variability than our measures of depression and stress, potentially leading to more non-significant findings in relation to anxiety symptoms. Finally, though we speculate that our findings are suggestive of an underlying temporal heterogeneity in the response of brain networks to MATT, we acknowledge that the evaluation of functional connectivity at rest, rather than on task, may introduce network bias.

\section{CONCLUSIONS}

In summary, MATT is a novel, noninvasive, self-administered home-use treatment designed to alleviate symptoms of anxiety disorders. MATT peripheral nerve stimulation appears to be associated with alterations of RSFC in the DMN after both acute and long-term administration. We found MATT-associated increases in connectivity between pain and anxiety ROls and the posterior DMN correlated with decreases in stress and depression symptoms over a four-week course of treatment. These results provide a foundation for understanding potential MATT mechanisms of therapeutic action and reflect an important first step in developing noninvasive alternative neuromodulation therapies that alleviate symptoms through alteration of brain connectivity. Although our results are promising, replication in a double-blinded randomized shamcontrolled trial is needed to evaluate potential test-retest or placebo effects.

\section{Authorship Statement}

Sean Hagberg, Linda L. Carpenter, and Jennifer Barredo conceived the basic idea and initiated, designed, and directed the study. Meghan A. Gonsalves and Jennifer Barredo wrote the manuscript. Eric Tirrell, Eugenia F. Kronenberg, Fatih Kokdere, Andrew M. Fukuda, and Quincy M. Beck collected the clinical and MRI data. Meghan A. Gonsalves, Quincy M. Beck, Jennifer Barredo, and Nicolas D. ladarola conducted the data quality control procedures and performed the statistical and data analyses. Meghan A. Gonsalves created the figures and tables. Meghan A. Gonsalves, Quincy M. Beck, Nicolas D. ladarola, Sean Hagberg, Linda L. Carpenter, and Jennifer Barredo provided input on data analysis and interpretation of results. Meghan A. Gonsalves, Quincy M. Beck,
Nicolas D. ladarola, Andrew M. Fukuda, Eric Tirrell, Fatih Kokdere, Eugenia F. Kronenberg, Sean Hagberg, Linda L. Carpenter, and Jennifer Barredo revised the manuscript. All authors read and approved the final manuscript.

\section{How to Cite This Article}

Gonsalves M.A., Beck Q.M., Fukuda A.M., Tirrell E., Kokdere F., Kronenberg E.F., ladarola N.D., Hagberg S., Carpenter L.L., Barredo J. 2022. Mechanical Affective Touch Therapy for Anxiety Disorders: Effects on Resting State Functional Connectivity.

Neuromodulation 2022; 25: 1431-1442.

\section{SUPPLEMENTARY DATA}

To access the supplementary material accompanying this article, visit the online version of Neuromodulation: Technology at the Neural Interface at www.neuromodulationjournal.org and at https:// doi.org/10.1016/j.neurom.2021.10.007.

\section{REFERENCES}

1. Strine TW, Mokdad AH, Balluz LS, et al. Depression and anxiety in the United States: findings from the 2006 behavioral risk factor surveillance system. Psychiatr Serv. 2008;59:1383-1390.

2. Ramsawh HJ, Stein MB, Belik SL, Jacobi F, Sareen J. Relationship of anxiety disorders, sleep quality, and functional impairment in a community sample. J Psychiatr Res. 2009;43:926-933.

3. Kessler RC, Chiu WT, Demler O, Merikangas KR, Walters EE. Prevalence, severity, and comorbidity of 12-month DSM-IV disorders in the National Comorbidity Survey Replication. Arch Gen Psychiatry. 2005;62:617-627.

4. Fluharty M, Taylor AE, Grabski M, Munafò MR. The association of cigarette smoking with depression and anxiety: a systematic review. Nicotine Tob Res. 2017;19: 3-13.

5. Ruscio AM, Hallion LS, Lim CCW, et al. Cross-sectional comparison of the epidemiology of DSM-5 generalized anxiety disorder across the globe. JAMA Psychiatry. 2017;74:465-475.

6. Baxter AJ, Vos T, Scott KM, Ferrari AJ, Whiteford HA. The global burden of anxiety disorders in 2010. Psychol Med. 2014;44:2363-2374.

7. Novak A, Burgess ES, Clark M, Zvolensky MJ, Brown RA. Anxiety sensitivity, selfreported motives for alcohol and nicotine use, and level of consumption. J Anxiety Disord. 2003;17:165-180.

8. Wittchen HU. Generalized anxiety disorder: prevalence, burden, and cost to society. Depress Anxiety. 2002;16:162-171.

9. Cuijpers P, Sijbrandij M, Koole SL, Andersson G, Beekman AT, Reynolds CF 3rd. Adding psychotherapy to antidepressant medication in depression and anxiety disorders: a meta-analysis. World Psychiatry. 2014;13:56-67.

10. Hofmann SG, Sawyer AT, Korte KJ, Smits JA. Is it beneficial to add pharmacotherapy to cognitive-behavioral therapy when treating anxiety disorders? A metaanalytic review. Int J Cogn Ther. 2009;2:160-175.

11. Taylor S, Abramowitz JS, McKay D. Non-adherence and non-response in the treatment of anxiety disorders. J Anxiety Disord. 2012;26:583-589.

12. Beekwilder JP, Beems T. Overview of the clinical applications of vagus nerve stimulation. J Clin Neurophysiol. 2010;27:130-138.

13. Gozani SN. Fixed-site high-frequency transcutaneous electrical nerve stimulation for treatment of chronic low back and lower extremity pain. J Pain Res. 2016;9:469479.

14. DeSantana JM, Walsh DM, Vance C, Rakel BA, Sluka KA. Effectiveness of transcutaneous electrical nerve stimulation for treatment of hyperalgesia and pain. Curr Rheumatol Rep. 2008;10:492-499.

15. Hein $E$, Nowak M, Kiess $O$, et al. Auricular transcutaneous electrical nerve stimulation in depressed patients: a randomized controlled pilot study. J Neural Transm (Vienna). 2013;120:821-827.

16. De Felice EA. Cranial electrotherapy stimulation (CES) in the treatment of anxiety and other stress-related disorders: a review of controlled clinical trials. Stress Med 1997;13:31-42.

17. Legon W, Rowlands A, Opitz A, Sato TF, Tyler WJ. Pulsed ultrasound differentially stimulates somatosensory circuits in humans as indicated by EEG and FMRI. PLoS One. 2012; 7: e51177. 
18. Gavrilov LR, Gersuni GV, Ilyinsky OB, Sirotyuk MG, Tsirulnikov EM, Shchekanov EE. The effect of focused ultrasound on the skin and deep nerve structures of man and animal. Prog Brain Res. 1976;43:279-292.

19. Priplata AA, Niemi JB, Harry JD, Lipsitz LA, Collins JJ. Vibrating insoles and balance control in elderly people. Lancet. 2003;362:1123-1124.

20. Chu Z, Thibault M, Fafara K, et al. Devices and Methods for Delivering Mechanical Stimulation to Nerve, Mechanoreceptor, and Cell Targets. Apex Neuro Holdings; 2021.

21. Woo SH, Ranade S, Weyer AD, et al. Piezo2 is required for Merkel-cell mechanotransduction. Nature. 2014;509:622-626.

22. McGlone F, Wessberg J, Olausson H. Discriminative and affective touch: sensing and feeling. Neuron. 2014;82:737-755

23. Marshall AG, McGlone FP. Affective touch: the enigmatic spinal pathway of the Ctactile afferent. Neurosci Insights. 2020;15:2633105520925072.

24. Vallbo A, Olausson H, Wessberg J, Norrsell U. A system of unmyelinated afferents for innocuous mechanoreception in the human skin. Brain Res. 1993;628:301-304.

25. Ploner M, Gross J, Timmermann L, Schnitzler A. Cortical representation of first and second pain sensation in humans. Proc Natll Acad Sci U S A. 2002;99:12444-12448.

26. Wall PD. On the relation of injury to pain. The John J. Bonica lecture. Pain 1979:6:253-264.

27. Tashima R, Koga K, Sekine M, et al. Optogenetic activation of non-nociceptive $A \beta$ fibers induces neuropathic pain-like sensory and emotional behaviors after nerve injury in rats. eNeuro. 2018:5:ENEURO0450-17.2018.

28. Colloca L, Ludman T, Bouhassira D, et al. Neuropathic pain. Nat Rev Dis Primers 2017;3:17002.

29. Ikeda R, Cha M, Ling J, Jia Z, Coyle D, Gu JG. Merkel cells transduce and encode tactile stimuli to drive $A \beta$-afferent impulses. Cell. 2014;157:664-675.

30. Olausson H, Wessberg J, Morrison I, McGlone F, Vallbo A. The neurophysiology of unmyelinated tactile afferents. Neurosci Biobehav Rev. 2010;34:185-191.

31. Zaki J, Ochsner KN, Hanelin J, Wager TD, Mackey SC. Different circuits for different pain: patterns of functional connectivity reveal distinct networks for processing pain in self and others. Soc Neurosci. 2007;2:276-291.

32. Zaki J, Davis JI, Ochsner KN. Overlapping activity in anterior insula during interoception and emotional experience. Neuroimage. 2012;62:493-499.

33. Avery JA, Drevets WC, Moseman SE, Bodurka J, Barcalow JC, Simmons WK. Major depressive disorder is associated with abnormal interoceptive activity and functional connectivity in the insula. Biol Psychiatry. 2014;76:258-266.

34. Paulus MP, Stein MB. Interoception in anxiety and depression. Brain Struct Funct. 2010;214:451-463.

35. Ploghaus A, Narain C, Beckmann CF, et al. Exacerbation of pain by anxiety is associated with activity in a hippocampal network. J Neurosci. 2001;21:9896-9903.

36. Zhuo M. Neural mechanisms underlying anxiety-chronic pain interactions. Trends Neurosci. 2016:39:136-145.

37. Sugawara A, Terasawa $Y$, Katsunuma R, Sekiguchi A. Effects of interoceptive training on decision making, anxiety, and somatic symptoms. Biopsychosoc Med. 2020;14:7.

38. Hanley AW, Mehling WE, Garland EL. Holding the body in mind: interoceptive awareness, dispositional mindfulness and psychological well-being. J Psychosom Res. 2017:99:13-20.

39. Haase L, Thom NJ, Shukla A, et al. Mindfulness-based training attenuates insula response to an aversive interoceptive challenge. Soc Cogn Affect Neurosci. 2016;11:182-190.

40. Farb N, Daubenmier J, Price CJ, et al. Interoception, contemplative practice, and health. Front Psychol. 2015;6:763.

41. Kraus T, Kiess O, HösI K, Terekhin P, Kornhuber J, Forster C. CNS BOLD fMRI effects of sham-controlled transcutaneous electrical nerve stimulation in the left outer auditory canal—a pilot study. Brain Stimul. 2013;6:798-804.

42. Frangos E, Komisaruk BR. Access to vagal projections via cutaneous electrical stimulation of the neck: fMRI evidence in healthy humans. Brain Stimul. 2017;10:19-27.

43. Kraus T, Hösl K, Kiess O, Schanze A, Kornhuber J, Forster C. BOLD fMRI deactivation of limbic and temporal brain structures and mood enhancing effect by transcutaneous vagus nerve stimulation. J Neural Transm (Vienna). 2007;114:1485-1493.

44. Fang J, Rong P, Hong Y, et al. Transcutaneous vagus nerve stimulation modulates default mode network in major depressive disorder. Biol Psychiatry. 2016;79:266273.

45. Otti A, Guendel H, Läer L, et al. I know the pain you feel-how the human brain's default mode predicts our resonance to another's suffering. Neuroscience. 2010;169:143-148.

46. Crone JS, Ladurner G, Höller Y, Golaszewski S, Trinka E, Kronbichler M. Deactivation of the default mode network as a marker of impaired consciousness: an fMRI study. PLoS One. 2011;6, e26373.

47. Spreng RN, Grady CL. Patterns of brain activity supporting autobiographical memory, prospection, and theory of mind, and their relationship to the default mode network. J Cogn Neurosci. 2010;22:1112-1123.

48. Kim H. Dissociating the roles of the default-mode, dorsal, and ventral networks in episodic memory retrieval. Neuroimage. 2010;50:1648-1657.

49. Wirth M, Jann K, Dierks T, Federspiel A, Wiest R, Horn H. Semantic memory involvement in the default mode network: a functional neuroimaging study using independent component analysis. Neuroimage. 2011;54:3057-3066.

50. Philippi CL, Tranel D, Duff M, Rudrauf D. Damage to the default mode network disrupts autobiographical memory retrieval. Soc Cogn Affect Neurosci. 2015;10:318326.
51. Pan J, Zhan L, Hu C, et al. Emotion regulation and complex brain networks: association between expressive suppression and efficiency in the fronto-parietal network and default-mode network. Front Hum Neurosci. 2018;12:70.

52. Raichle ME. The brain's default mode network. Annu Rev Neurosci. 2015;38:43304447.

53. Zhao XH, Wang PJ, Li CB, et al. Altered default mode network activity in patient with anxiety disorders: an fMRI study. Eur J Radiol. 2007;63:373-378.

54. Tao Y, Liu B, Zhang X, et al. The structural connectivity pattern of the default mode network and its association with memory and anxiety. Front Neuroanat. 2015:9:152.

55. Sheline $\mathrm{Yl}$, Barch DM, Price $\mathrm{J}$, et al. The default mode network and self-referential processes in depression. Proc Natl Acad Sci U S A. 2009;106:1942-1947.

56. Wise T, Marwood L, Perkins AM, et al. Instability of default mode network connectivity in major depression: a two-sample confirmation study. Transl Psychiatry. 2017;7:e1105.

57. Sylvester CM, Corbetta M, Raichle ME, et al. Functional network dysfunction in anxiety and anxiety disorders. Trends Neurosci. 2012;35:527-535.

58. Lowe MJ, Dzemidzic M, Lurito JT, Mathews VP, Phillips MD. Correlations in lowfrequency BOLD fluctuations reflect cortico-cortical connections. Neuroimage. 2000;12:582-587.

59. van den Heuvel MP, Hulshoff Pol HE. Exploring the brain network: a review on resting-state fMRI functional connectivity. Eur Neuropsychopharmacol. 2010;20:519-534.

60. Dennis EL, Gotlib IH, Thompson PM, Thomason ME. Anxiety modulates insula recruitment in resting-state functional magnetic resonance imaging in youth and adults. Brain Connect. 2011;1:245-254.

61. Zhang S, Wu W, Huang G, et al. Resting-state connectivity in the default mode network and insula during experimental low back pain. Neural Regen Res. 2014;9:135-142.

62. Kucyi A, Moayedi M, Weissman-Fogel I, et al. Enhanced medial prefrontal-default mode network functional connectivity in chronic pain and its association with pain rumination. J Neurosci. 2014;34:3969-3975.

63. Duval ER, Javanbakht A, Liberzon I. Neural circuits in anxiety and stress disorders: a focused review. Ther Clin Risk Manag. 2015;11:115-126.

64. Feurer C, Suor JH, Jimmy J, et al. Differences in cortical thinning across development among individuals with and without anxiety disorders. Depress Anxiety. 2021:38:372-381.

65. World Health Organization. The ICD-10 Classification of Mental and Behavioural Disorders: Clinical Descriptions and Diagnostic Guidelines. Geneva: World Health Organization; 1992.

66. Sheehan DV, Lecrubier $Y$, Sheehan $\mathrm{KH}$, et al. The mini-international neuropsychiatric interview (M.I.N.I.): the development and validation of a structured diagnostic psychiatric interview for DSM-IV and ICD-10. J Clin Psychiatry. 1998;(59 suppl 20):22-33; quiz 34-57.

67. Spitzer RL, Kroenke K, Williams JB, Löwe B. A brief measure for assessing generalized anxiety disorder: the GAD-7. Arch Intern Med. 2006;166:1092-1097.

68. Cohen S, Kamarck T, Mermelstein R. A global measure of perceived stress. J Health Soc Behav. 1983;24:385-396.

69. Beck AT, Ward CH, Mendelson M, Mock J, Erbaugh J. An inventory for measuring depression. Arch Gen Psychiatry. 1961;4:561-571.

70. Parkitny L, McAuley J. The depression anxiety stress scale (DASS). J Physiother. 2010;56:204.

71. Mehling WE, Price C, Daubenmier JJ, Acree M, Bartmess E, Stewart A. The multidimensional assessment of interoceptive awareness (MAIA). PLoS One. 2012;7, e48230.

72. Whitfield-Gabrieli S, Conn Nieto-Castanon A. a functional connectivity toolbox for correlated and anticorrelated brain networks. Brain Connect. 2012;2:125-141.

73. Power JD, Barnes KA, Snyder AZ, Schlaggar BL, Petersen SE. Spurious but systematic correlations in functional connectivity MRI networks arise from subject motion. Neuroimage. 2012;59:2142-2154.

74. Ciric R, Wolf DH, Power JD, et al. Benchmarking of participant-level confound regression strategies for the control of motion artifact in studies of functional connectivity. Neuroimage. 2017;154:174-187.

75. Behzadi Y, Restom K, Liau J, Liu TT. A component based noise correction method (CompCor) for BOLD and perfusion based fMRI. Neuroimage. 2007;37:90-101.

76. Yarkoni T, Poldrack RA, Nichols TE, Van Essen DC, Wager TD. Large-scale automated synthesis of human functional neuroimaging data. Nat Methods. 2011;8:665-670.

77. Fischl B. FreeSurfer. Neuroimage. 2012;62:774-781.

78. Dale AM, Fischl B, Sereno MI. Cortical surface-based analysis. I. segmentation and surface reconstruction. Neurolmage. 1999;9:179-194.

79. Fischl B, Dale AM. Measuring the thickness of the human cerebral cortex from magnetic resonance images. Proc Natl Acad Sci U S A. 2000;97:11050-11055.

80. Fischl B, Salat DH, Busa E, et al. Whole brain segmentation: automated labeling of neuroanatomical structures in the human brain. Neuron. 2002;33:341-355.

81. Fischl B, Sereno MI, Dale AM. Cortical surface-based analysis. II: inflation, flattening, and a surface-based coordinate system. Neuroimage. 1999;9:195-207.

82. Fischl B, Sereno MI, Tootell RB, Dale AM. High-resolution intersubject averaging and a coordinate system for the cortical surface. Hum Brain Mapp. 1999;8:272-284.

83. Fischl B, van der Kouwe A, Destrieux C, et al. Automatically parcellating the human cerebral cortex. Cereb Cortex. 2004;14:11-22.

84. Reuter M, Rosas HD, Fischl B. Highly accurate inverse consistent registration: robust approach. Neuroimage. 2010;53:1181-1196. 
85. Desikan RS, Ségonne F, Fischl B, et al. An automated labeling system for subdividing the human cerebral cortex on MRI scans into gyral based regions of interest. Neuroimage. 2006:31:968-980.

86. Liao W, Chen H, Feng $\mathrm{Y}$, et al. Selective aberrant functional connectivity of resting state networks in social anxiety disorder. Neuroimage. 2010;52:1549-1558.

87. Lanius RA, Bluhm RL, Coupland NJ, et al. Default mode network connectivity as a predictor of post-traumatic stress disorder symptom severity in acutely traumatized subjects. Acta Psychiatr Scand. 2010;121:33-40.

88. Andrews-Hanna JR, Reidler JS, Sepulcre J, Poulin R, Buckner RL. Functionalanatomic fractionation of the brain's default network. Neuron. 2010;65:550-562.

89. Laing PAF, Burns N, Baetu I. Individual differences in anxiety and fear learning: the role of working memory capacity. Acta Psychol (Amst). 2019;193:42-54.

90. Nakajima M, Takano K, Tanno Y. Adaptive functions of self-focused attention: insight and depressive and anxiety symptoms. Psychiatry Res. 2017;249:275-280.

91. Lu C, Yang T, Zhao H, et al. Insular cortex is critical for the perception, modulation, and chronification of pain. Neurosci Bull. 2016;32:191-201.

92. Uddin LQ. Salience processing and insular cortical function and dysfunction. Nat Rev Neurosci. 2015;16:55-61.
93. Wiech $\mathrm{K}$, Lin CS, Brodersen $\mathrm{KH}$, Bingel $\mathrm{U}$, Ploner $\mathrm{M}$, Tracey I. Anterior insula integrates information about salience into perceptual decisions about pain. J Neurosci. 2010;30:16324-16331.

94. Ellard KK, Zimmerman JP, Kaur N, et al. Functional connectivity between anterior insula and key nodes of frontoparietal executive control and salience networks distinguish bipolar depression from unipolar depression and healthy control subjects. Biol Psychiatry Cogn Neurosci Neuroimaging. 2018;3:473-484.

95. Menon V, Uddin LQ. Saliency, switching, attention and control: a network model of insula function. Brain Struct Funct. 2010;214:655-667.

96. Allen M, Fardo F, Dietz MJ, et al. Anterior insula coordinates hierarchical processing of tactile mismatch responses. Neuroimage. 2016;127:34-43.

97. Gallese V. Embodied simulation: from neurons to phenomenal experience. Phe nomenol Cogn Sci. 2005;4:23-48.

98. Bornemann B, Herbert BM, Mehling WE, Singer T. Differential changes in selfreported aspects of interoceptive awareness through 3 months of contemplative training. Front Psychol. 2015;5:1504.

99. Lieberman MD, Cunningham WA. Type I and Type II error concerns in fMRI research: re-balancing the scale. Soc Cogn Affect Neurosci. 2009;4:423-428. 